\title{
A Unique Approach to Implement Semantic Web
}

\author{
Vandana Bharti \\ M.Tech Scholar (CSE) \\ Tula's Institute, Dehradun, India
}

\author{
Madhu Kirola \\ Assistant Professor (CSE Department) \\ Tula's Institute, Dehradun, India
}

\begin{abstract}
Information discovery mechanisms of web services in the UDDI registries currently are not up to mark of Industry standards. UDDI provides the interface keyword and taxonomy based search mechanism. UDDI refers exact keyword matching discovery mechanism. If the consumer searches for any keyword like "Apartment", the service provider registered under the tag "flat" will not be shown to the end user. Additionally there is possibility that existing taxonomies might not be appropriate enough, extracting very long result lists may not really help. The main issue is lack of semantics. XML Topic Maps as a representative of Semantic Web may help to improving the discovery mechanism. The present work defines the stated problem and as of my knowledge provides a new solution to the current problem. Our main aim is to show how XML Topic Maps can be used as tool to represent and organize knowledge out of specific domain, in our case bioinformatics. XML Topic Maps will give the advantages to requestor, who needs to find some location of unknown web service or resource. A prototypical Topic Map a sub domain of Gene Ontology. It will be developed and after implementation it will be shown that how the representation of knowledge can be linked to UDDI. The XML Topic Map can be navigated in different ways. It reaches the aim and discovers previously unknown resource. XTM can be used for building knowledge base mechanism. To navigate through the user need to use a particular term. Once a unique term is assigned a large number of external useful information resources is also added to it. The most important aspect is that due to the continuous evolution of Gene Ontology the XTM documentation has to be maintained permanently. At the end it was concluded that refering directly to the location of WSDN file and doing job without UDDI will not be feasible.
\end{abstract}

\section{Keywords}

XLM, UDDI, XTM, Semantic web, Gene Ontology, topic map.

\section{INTRODUCTION}

Web Technology is growing at very faster rate and the requirement of searching the web pages need to be more efficient. Semantic web exploit some of the basic concept of web technology and try to improves upon the existing web services[4]. At present the web is full of various web pages and data. The current system use keywords to search across the web pages and web system. This entire system is a meaningless as the data searched through the existing technologies comes out to be redundant sometimes. Semantic web empowers and improvise the existing web search technology so as to express all the resources on the web. Ontologies plays a major role in the field of Semantic Web. There are some description languages that used for defining an ontology, like OWL (Web Ontology Language), RDF (Resource Description Framework) and RDFS (Resource Description Framework Schema)[4]. On the world wide web,
$\mathrm{RDF}$ is a language describing relation and resources between them. The semantic web and web services can be moved together so the search services can be made more consistent and efficient.

\section{REAL TIME SCENARIO}

Everyone is hunting down a particular Web Service out of the organic area. This paper will show how the discovery problems present in the existing system can be solved by an XTM solution. These prompt a change of the pursuit process. Such type an answer is new, and subsequently, as far as anyone is concerned, in this way there is no execution.

We take after a predefined technique. This is the motivation behind why the production of a metaphysics must be overall arranged so as to dodge issues amid the improvement and to guarantee that the undertaking is a win.

"There is nobody remedy approach to model an area - there are constantly practical options." One such option, case in point, was produced at the Stanford University. It depicts a conceivable approach to create ontologies with the Protégé programming instrument.

Be that as it may, such a strategy to model a space speaks to a sort of venture administration with diverse breakthroughs. In any case before we change to the usage segment, some more learning is required. The extent that the careful investigation considers the organic area, the following segment will be concerned with natural ontologies. This is essential, in light of the fact that by and large it is imagined to reuse ontologies.

\subsection{Biological Ontologies}

Ontologies are getting more weight as a focal segment of numerous applications. In bioinformatics (the control of applying figuring to sub-atomic science), ontologies picked up force in the previous years, inspired by the need to reliably get to, depict, impart and control the in excess of 500 databases in standard utilization. The complex natural information put away in bioinformatics databases requires regularly the expansion of learning to tag and oblige the qualities held in those databases[10]. In addition, numerous researcher are working by applying former information to an obscure substance. Ontologies are for the most part seen as a guaranteeing system for communicating and offering the atomic science group's testing rich, dynamic and complex information. While the sum and multifaceted nature of that information builds, the need to endeavor it effectively and successfully develops[1]. Note that ontologies assume a vital part likewise for other new fields (nanotechnology, and so on.); for our situation bioinformatics has been picked as 'universe of talk'.

\subsection{Linking the XLM topic map to UDDI}

It is clear that a learning representation framework must be connected some way or another to the UDDI registry with a 
specific end goal to be helpful. The accompanying subsections are managing this issue. In the majority of these possible alternatives the XML Topic Map goes about as a point/semantic layer over the UDDI registry.

\subsection{Ontology to UDDI}

It was said prior that the semantic issue as for UDDI was additionally distinguished by the UDDI group.

Figure 1 demonstrates the first plausibility of how a philosophy could be connected to a UDDI element. A metaphysics component is referencing a UDDI element. The uniqueness of the UUID key (Universal Unique Identifier) empowers the linkage between a learning representation and a UDDI substance[9].

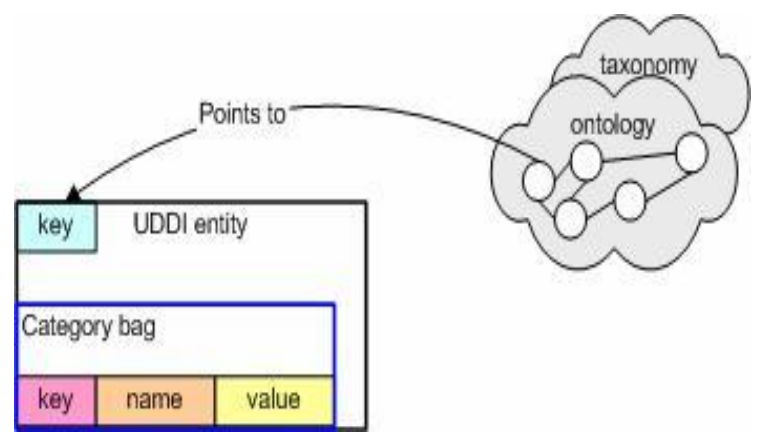

Figure 1: Ontology points to UDDI entity

\section{PROTOTYPICAL IMPLEMENTATION}

Before Now we define a step by step process for developing a Topic Maps. This methodology consists of following seven steps:
A: Defining the aim
B: Taking additional possible prerequisites
C: Reusing existing ontologies or defining new ones
D: Setting up relevant topics
E: Assigning information resources
F: Setting up associations between the topics
G: Testing the topic map

Now, the following subsections tackle these steps more detailed and describe what actions and questions have been considered during each step.

\subsection{Defining the Aim}

The primary step ought to incorporate musings about the extent of the expected work. The essential point, regarding this work, is to enhance the revelation procedure of Web Services in the zone of bioinformatics. Along these lines, the fundamental purchasers of such a cosmology will apparently be scientists looking for at one time obscure assets (fundamentally in type of Web Services) on the web.

Also to the improvement of a philosophy, it must be indicated how this metaphysics helps attaining the objective, i.e. to demonstrate how the philosophy can be utilized through sufficient route for spotting particular assets out of a certain area.

\subsection{Talking Additional Possible Prerequisites}

The second venture in this approach is likewise critical, on the grounds that here it ought to be chosen how the learning base must be produced. The prototypical subject guide will be produced physically in a basic content manager with the assistance of the LTM documentation for reasons prior portrayed in the postulation.

\subsection{Reusing Existing Ontologies or Defining New Ones}

The inquiry whether to reuse existing ontologies or to characterize new ones must be replied right now. It ought not be belittled that the advancement of another information representation requires the ramifications of area masters and is very lengthy.

Ontologies claim to speak to the learning out of a particular area and in this manner imagine to be reused. The Gene Ontology, for example, is a broadly utilized cosmology and in this way a trustful source to be reused. In spite of the wording, the Gene Ontology is not a metaphysics, however an organized controlled vocabulary. This structure is comprised of "hubs" and "edges", which relate in point maps to "themes", individually "affiliations".

The wording of the Gene Ontology is utilized to clarify quality items regarding three qualities: the sub-atomic capacities of these items, the larger amount organic methods in which they are included and the cell parts where they can be found. These three traits develop three chains of command, which are autonomous of one another, that imply that no connections between terms from discrete orders exist. In the connection of this proposal we will focus on the last property, in particular cell part and out of it the subset virion.

\subsection{Setting up relevant topics}

A mid this step we need to set up the themes for our subject guide. The Gene Ontology comprises of comprehensible terms. Every hub in the chart is a characteristic dialect term, and for very nearly all terms, a regular dialect definition is given.

It was specified prior that a "hub" speaks to a "subject". So it is not difficult to situated up the pertinent subjects and this is one of the primary preferences of reusing effectively existing and settled ontologies. In segment 4.7 it was exemplified how points in LTM are made and look like.

\subsection{Assigning information resources}

Area 3.1 indicated how a cosmology could be connected to UDDI. A passage in the UDDI registry speaks to a data asset. It was additionally said that every UDDI substance is extraordinarily recognized through a key (UUID). Subsequently it is not hard to appoint such a data asset.

Anyhow this prototypical execution won't allude just to UDDI substances. Segment 3.3 portrayed that a theme can allude additionally specifically to the depiction of an administration. Both areas will be considered for the execution and furthermore other data assets will be prototypically doled out. 
Such assets could for example be figures, downloaddocuments from a ftp-server, connections to significant investigative distributions, and so on.

Consequently the result can be seen as a "complete" information base for researcher. This learning base has a much greater usefulness than the title of this postulation guarantees, on the grounds that it doesn't allude just to UDDI and Web Services, however to assets all in all. Note that in the prototypical execution imaginary areas of data assets were utilized. This can be accomplished through various types of "event sorts". Figure 2 shows what sort of assets can commendable be alloted to a theme of a metaphysics communicated in XTM.

\subsection{Setting up association between the topics}

The motivation behind this step is to situated up relationship between the points created in step 4. Each of the three chains of command of the Gene Ontology is built from a mixture of scientific classification and partonomy. Thusly each one edge in the chart is either a "will be an" or an "a piece of" connection.

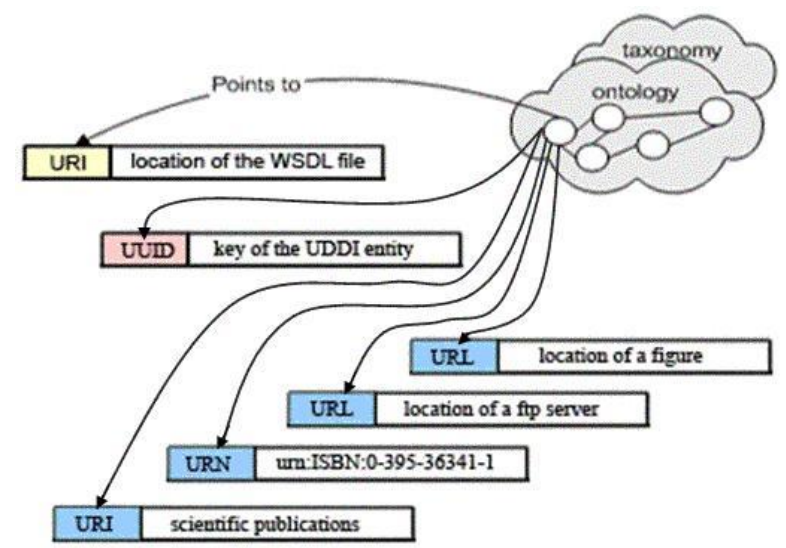

Figure 2: Ontology, a complete knowledge base pointing to different types information resources in one domain

Remark that the suggested solution becomes an alternative to the UDDI in this way.

The offices offered by LTM grant us to speak to the aforementioned relations in a delicate manner. Case in point, in the Gene Ontology the edge between the expressions "viral capsid" and "virion" is an "a piece of"-connection. This is spoken to in LTM like:

$$
\text { part-of\{viral_capsid, virion\} }
$$

\subsection{Testing the topic map}

This is the last venture in the improvement of the theme map. The extent that our LTM - archive was hand-coded, it is extremely conceivable to have some writing slips. Keeping in mind the end goal to demonstrate whether the data contained in our subject guide is reliable and compelling, we utilized the Ontopia Omnigator for debugging our theme map[8]. On the off chance that there are mistakes, the Omnigator does not stack the theme map, however it gives significant indications in regards to the blunder and its area in the LTM archive.

After the LTM was stacked effectively, the fare usefulness was connected and the subject guide was sent out to the XTM
1.0 punctuation. For space reasons the XTM report is not joined to the theory, yet included a CD sticking the postulation.

\begin{tabular}{|l|}
\hline Index of Topic Maps \\
- bio.Itm \\
- bio.xtm \\
- cell.Itm \\
- cell.xtm \\
\hline
\end{tabular}

Figure 3: topic maps in different formats

\section{NAVIGATION THE TOPIC MAP}

After the development we will be shown here how our topic map can be navigated. Omnigator offers various ways in doing so:

1) Visual navigation through the Vizigator

2) Link navigating the Omnigator HTML pages

3) tolog - a language for querying topic maps.

\subsection{Link Navigating the Omnigator HTML Pages}

Omnigator utilizes a straightforward customer server construction modeling focused around a standard http convention. The application on the server side runs on a Tomcat web server. It peruses and composes point maps and creates HTML pages on the fly. On the customer, a standard web program gets these HTML pages and presentations a perspective of some share of the theme map. This perspective is rich in connections, manufactured from the information structures that constitute the subject guide. Each one time the client clicks on a connection, a solicitation is sent to the server application, bringing about another set of data concentrated from the theme map [11].

In Figure 4 the connection for the GO term Viral genome was clicked and all data on that point is shown.

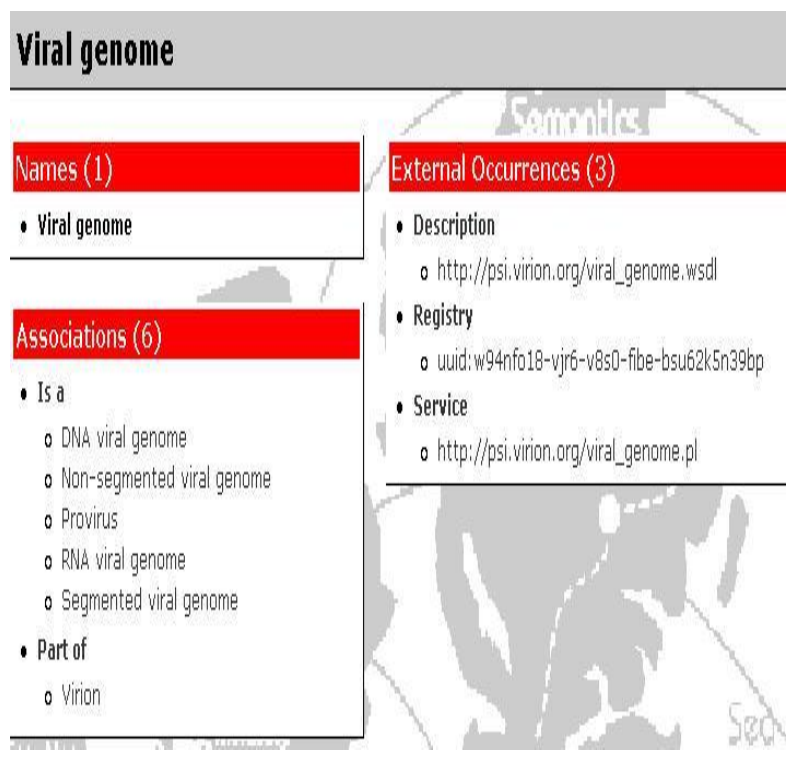

Figure 4 : Navigating Omnigator HTML Pages 


\subsection{Visual Navigation through the Vizigator}

The Vizigator is gotten to by means of the Vizigate module and gives the client an extra technique for theme map route through graphical scanning. The Vizigator empowers the client to have a more broad view on the theme map, on the grounds that it is conceivable to pick and access the craved level of profundity. In the upper left corner of Figure 6 we can fill in the case the sought number of levels to be shown (for our situation - 3). Little red boxes with numbers demonstrate further profundities in the representation. In the same figure the two connections/affiliations can be recognized through distinctive colors; pink edges stand for the "a piece of" and purple edges stand for the "is-an" affiliations. The begin theme is constantly shown in yellow and speaks to the subject where the client generally begins her/his manual perusing from. All or piece of a theme name must be filled in the pursuit box and the hit will flicker in green. Case in point, we looked for the GO-term DNA viral genome, and the result is similar to indicated in the accompanying figure:

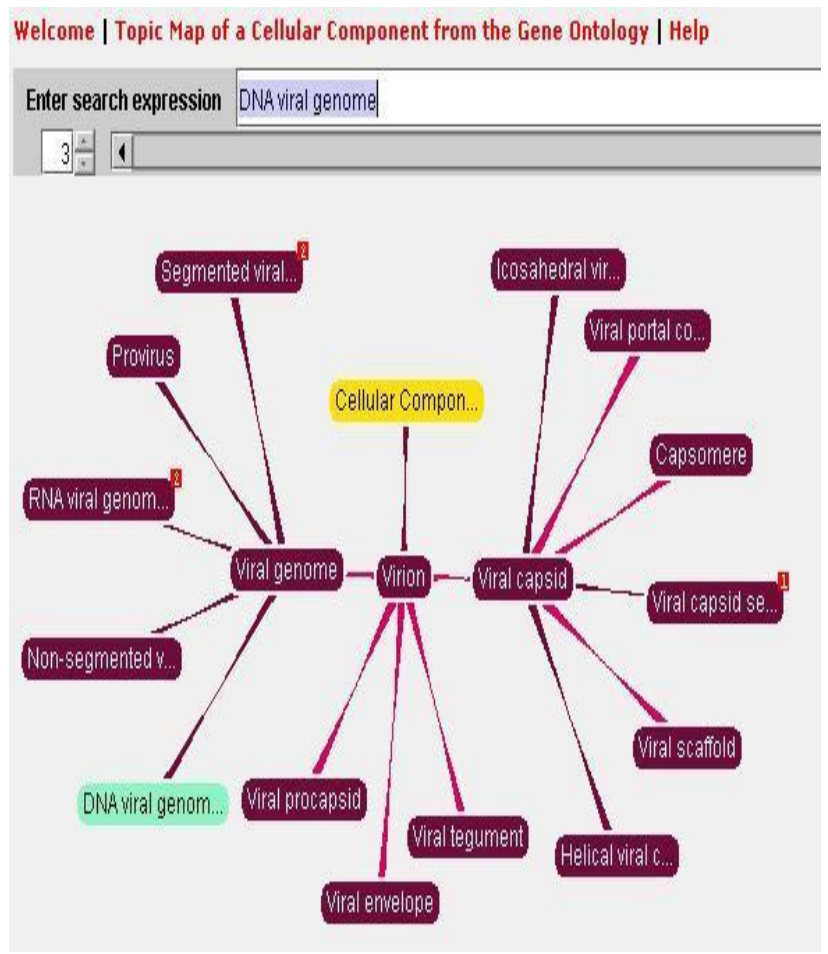

Figure 5: Graphically Navigating the Cellular Component of GO

Presently, by right-clicking on the result (or the theme of yearning), we are getting to the insights about the outside events Figure 6).

\subsection{Tolog - A Language for Querying Topic Maps}

"tolog is a rationale based inquiry dialect, which implies that the essential operation is for the client to ask tolog in which cases a certain attestation holds genuine, and tolog will then react with all the sets of values that make the statement genuine" [12].

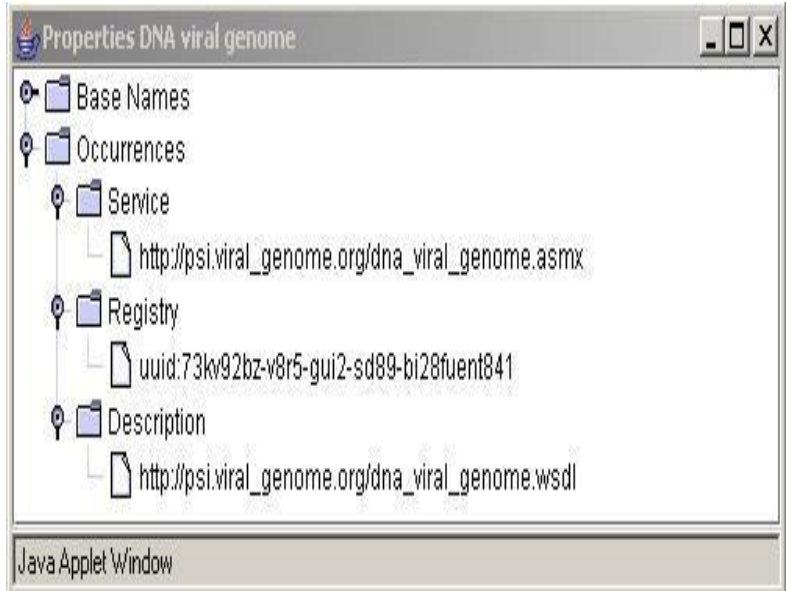

Figure 6: Properties with occurrences for a specific topic

Returning to the case of last segment, where we were occupied with outside data sources (events) about the GO-term DNA viral genome, a tolog order would look like:

event (Dna_viral_genome, \$occ)?

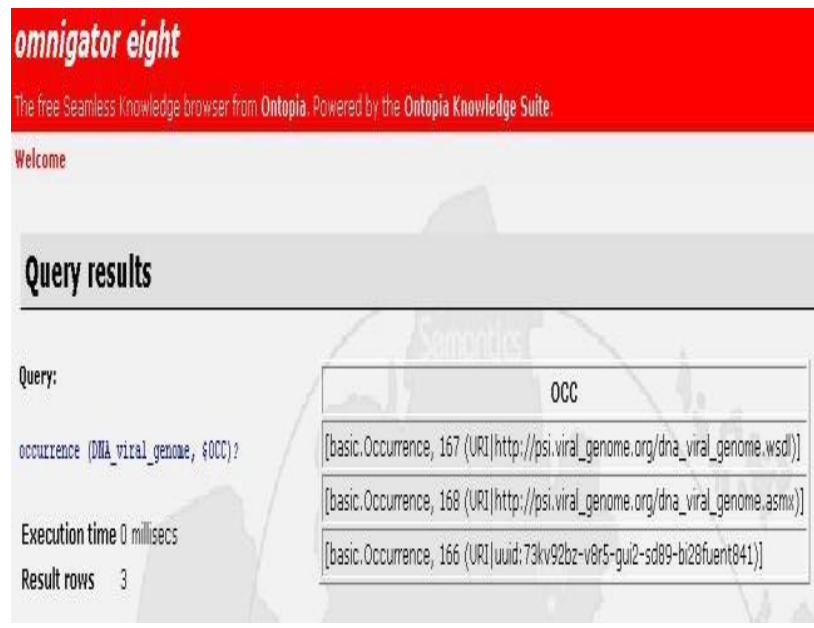

Fig 7: tolog query results

\section{XTM IN COMPARISON TO ALTERNATIVE TECHNOLOGIES}

In spite of the fact that is not generally a sense in contrasting things, for our situation it might be of enthusiasm to look quickly at Topic Maps and OWL (as superseding RDF) keeping in mind the end goal to have a superior thought how these intriguing innovations "contend" one another, however taking care of really diverse issues.

Above all else, Topic Maps and RDF have diverse starting points: they are originating from two standard associations ISO, individually $\mathrm{W} 3 \mathrm{C}$. This was possibly the point which decided some insufficient educated gatherings to see them as contenders[5]. Both innovations case to approach facilitating the issue of infoglut, utilizing learning representation methods. In Topic Maps, the key-idea is the theme, made from the need

of discussing subjects, while in RDF, the asset lies in the focal point. It can then be said that the highlighted terms are assuming the same part. Simply that Topic Maps are learning representation from the viewpoint of people, while RDF from the point of view of machines [2]. 
The themes have in Topic Maps qualities - names, events and relationship; in RDF, such things don't exist. In Topic Maps, the subjects out of whom points are made, with a specific end goal to make articulations about them, can be addressable or non-addressable. The addressable subjects are recognized by Uris and the non-addressable ones - by the Uris of the "subject pointers". The PSIs in Topic Maps encourage interoperability crosswise over applications; in RDF such property does not exist. In RDF they are simply assets, distinguished by Uris, yet no other refinement. On the other side, the way that Psis are laying on Uris facilitates the interoperability for both advances.

In the event that we take a gander at affiliations, the evident perspective is that in Topic Maps we can have more than two "players", as RDF has. At the end of the day, in Topic Maps, more than two parts can happen in an affiliation. In the event that we stretch out now the perspective to OWL, in regards to scientific categorizations, Topic Maps offer a more straightforward approach to express same things that OWL could do, while OWL gives semantic legitimacy[6]. Additionally, a portion of the semantic establishments are indistinguishable in that Topic Maps do help class/subclass affiliations, despite the fact that the class demonstrating in Topic Maps is not very solid. Point Maps depend generally on name-based arrangement (and uniting), while OWL classes have vigorous properties, set theoretics and mapping. Nonetheless, OWL and XTM are serializations with which one can send data and have it landed in a decipherable structure at the flip side, regardless of which wire is utilized. Both bring to the table a basic methodology model and semantics. XTM makes a moderate ontological responsibility to question important to catch themes and subjects. To this, OWL includes a few expressiveness, so it can be exploited supplementing the innovations [3].

At last, it can be said that XML Topic Maps discovered their spot in the Semantic Web world and that there is no genuine rivalry between the specified innovations.

\section{RESULTS}

The Web Services and Semantic Web are two new exciting research field. In the centre of this work lays the concept of Topic Maps, more properly of XML Topic Map. Here we can be used for navigating enormous amount of information. XTM do not support formal reasoning, but are excellent aid for helping people lost in information space. In the world of Web Services, UDDI play the role of the yellow pages, but UDDI does not realize what it aim, because search capability within the registry are quite limited. The purpose of this paper was to improve the process of services discovery by moving to more semantics. For this retrieval problem, we exploited the capability of XML Topic Maps. There exist a couple of efforts in combining Web Services with the Semantic Web in order to achieve a better search for Web Services. To my knowledge, currently such kind of implementation does not exist, with the aim to improve service discovery.

When we talking about XTM, we talk simultaneously about the organizing knowledge, more specifically about term like taxonomy and ontology. Those construct consists of semantic [13] term and relation, whole communities agreed on. For this reason we used a small part of the Gene Ontology as a basis for our prototype in our case study.

\section{CONCLUSION}

This research work introduced first the purpose of the case study and described briefly a few biological ontologies. This was shown that a topic map solution that can act as a complete knowledge base with links to different type information resources. This, of course, implies that our initial aim was reached, namely to base the discovery of Web Services on Semantic Web technologies.

\section{REFERENCES}

[1] An Introduction to Gene Ontology, [online] available at : www.geneontology.org/GO.doc.html, edited: 2005-0111, visited: 2005-01-14

[2] Pepper S., Ten Theses on Topic Maps and RDF, [online] available at www.ontopia.net/topicmaps/materials/rdf.html , edited: 2003-04-15, visited: 2004-12-14

[3] ontolog forum, "Topic Maps and OWL", [online] available at www.ontolog.cim3.net/forum/ontologforum/2004-01/msg00014.html , edited: 2004-01-02, visited: 2005-01-17

[4] Antoniou G., van Harmelen F., A Semantic Web Primer,London, MIT Press, 2004, ISBN 0-262-01210-3

[5] Biezunski M., Newcomb S. R., XML Topic Maps: Finding Aids for the Web, 2001, [online] available at: www.coolheads.com/SRNPUBS/ieee-mm-topicmapsarticle.pdf

[6] Carlstedt, A., Nordborg M., An evaluation of Topic Maps, A Master's Thesis in Computational Linguistics, Göteborg University, May 2002

[7] Garshol L. M., 24re G., Topic Maps - Data Model, 2003, [online], available at http://www.isotopicmaps.org/sam/sam-model/ , edited: 2005-01-17, visited 2005-05-12

[8] Fensel D., ontologies: A Silver Bullet for KnowledgeManagement and Electronic Commerce, Berlin, Springer, 2004, ISBN 3-540-00302-9

[9] Freese E., "SemanText", in: Park J., Hunting S., XML TopicMaps, Creating and using Topic Maps for the Web, Boston, Addison Wesley, 2002, ISBN 0-20174960

[10] Garshol L. M., private mail on 2005-01-19

[11] The Ontopia Omnigator "User's Guide" [online] available at www.ontopia.net/omnigator/userguide.html, edited: 2004-12-09, visited: 2005- 01-15

[12] tolog - Language Tutorial, [online] available at : www.ontopia.net/omnigator/docs/query/tutorial.html, edited: 2004-12-08, visited: 2005-03-11 